\title{
基于兴趣点的北京市住宅生活便利度指数研究
}

\author{
李健，张松海* \\ (清华大学计算机科学与技术系 北京 100084) \\ (shz@tsinghua.edu.cn)
}

\begin{abstract}
摘 要: 住宅的生活便利度指数指住宅居民在一定范围内可利用的设施种类与数量, 是城市宜居度的重要指标. 兴 趣点(point of interest, POI)数据是一组包含物理实体属性的地理坐标点, 具有数据种类详尽、粒度精细、范围广和时 效性强的优点. 基于高德 POI 数据, 对北京市城区住宅的生活便利度指数进行研究, 提出面向地铁出行的各向异性 距离度量方法，根据构建的生活便利度指数指标体系将住宅分为优质、良好、中等和待改进 4 个等级. 各等级住宅在 城区中的空间分布结果表明, 考虑地铁出行后, 对住宅的生活便利度指数度量更为合理.
\end{abstract}

关键词：兴趣点数据; 生活便利度指数; 各向异性; 距离度量

中图法分类号: TP391.41 DOI: 10.3724/SP.J.1089.2021.18539

\section{Research on Beijing Residential Convenience Index Based on Point of Interest}

Li Jian and Zhang Songhai*

(Department of Computer Science and Technology, Tsinghua University, Beijing 100084)

\begin{abstract}
Residential convenience index refers to the types and number of facilities available to residential residents within a certain range, which is a significant indicator for urban livability. Point of interest (POI) data is a set of geographic coordinate points containing physical entity attributes. It has the advantages of detailed data types, fine granularity, wide range and strong timeliness. Based on the POI data of Amap, the residential convenience index of buildings in Beijing's urban areas is studied, and an anisotropic distance measurement method for subway travel is proposed. According to the constructed index system of living convenience index, houses are divided into excellent, good, moderate and poor levels. The results of the spatial distribution of various levels of residences in the urban area show that after taking subway trips into account, the convenience index measures of all levels of dwellings are more reasonable.
\end{abstract}

Key words: POI data; convenience index; anisotropy; distance measure

随着我国经济的不断发展和城市化的不断推 进, 越来越多的人口进人城市, 人们对城市的人居 环境关注度逐渐提升. 长期以来, 城市管理者以发 展生产为城市规划目标, 导致城市区域功能过于 集中, 公共设施空间分布不均匀, 与以人为本的城 市发展理念不符. 随着人民物质文化的需求日益 增长, 如何合理地增加各区域的公共服务设施, 使
居民生活更为美好, 成为城市管理者迫切需要解 决的问题 ${ }^{[1]}$.

生活便利度的研究起源于日本, 20 世纪 5060 年代, 随着工业化和城市化的飞速发展, 日本 产生了一系列的人居环境问题, 因此日本政府提 出了生活圈的概念, 通过规划公共设施引导实现 各个区域的平衡发展. 我国也较早地引人了生活

收稿日期: 2020-08-02; 修回日期: 2020-09-30. 基金项目：国家自然科学基金(61832016, 61772298). 李健(1997一), 男, 硕士研 究生, 主要研究方向为图像处理、人工智能; 张松海(1978一), 男, 博士, 副教授, 博士生导师, CCF 会员, 论文通讯作者, 主要研究方 向为图像、视频处理、计算机图形学. 
圈的概念, 但更多的是作为城市规划的参考. 随着 城市化进程推进, 引起了城市发展问题, 生活圈生 活便利度指数才引起了更多学者的关注. 已有的 生活圈生活便利度指数研究多是基于居民问卷调 查，如李萌 ${ }^{[2]}$ 基于 200 份居民问卷对上海市“15 分 钟社会生活圈”进行居民需求分析并提出规划对 策. 但问卷调查研究存在调查成本高、数据样本少 和调查范围小的缺点, 使其难以拓展到研究整个 城市的住宅便利度指数研究.

\section{1 相关工作}

大数据的发展为城市住宅便利度指数的研究 提供了新方法, 兴趣点(point of interest, POI)数据 是一组包含物理实体属性的地理坐标点, 以其数 据类型详尽、覆盖范围广和数据时效性强的优点引 起了许多学者的关注. 基于 POI 数据, 吴康敏等 ${ }^{[3]}$ 对广州市的多类型商业中心进行识别和空间分布 研究; 林清等 ${ }^{[4]}$ 划分了北京市的商业中心等级; 许 泽宁等 ${ }^{[5]}$ 提出了对城市建成区的边界识别的方法; 李江苏等 ${ }^{[6]}$ 对郑东新区的服务业空间进行聚类研 究, 为区域的产业结构提出优化方向; 赵云丹等 ${ }^{[7]}$ 对杭州市中心城区的公共设施空间分布进行研究, 为城市规划提供参考. 丁娟等 ${ }^{[8]}$ 基于人境旅客 POI 数据, 对其热门旅游景点的空间分布进行研究. $\mathrm{Lu}$ 等 ${ }^{[9]}$ 利用深度神经网络, 为社交用户提供新的 POI 推荐. Santos 等 ${ }^{[10]}$ 利用用户的 POI 信息, 为其提供 旅游目的地推荐. 近年来也有基于 POI 数据对生 活圈生活便利度指数的积极探索, 如崔真真等 ${ }^{[11]}$ 对北京、天津、上海和广州的城市生活便利度指数 进行研究; 赵彦云等 ${ }^{[12]}$ 和张波等 ${ }^{[13]}$ 对北京市的“15 分钟社区生活圈”进行定量研究, 为北京市的城市 空间优化提供科学依据.

现有的基于 POI 数据统计分析的研究方法主 要使用二维欧几里得度量作为方式度量. 但在北 京等超大规模城市中, 地铁是一种主要的交通出 行方式，在计算居民可利用设施中是一种重要的 考虑因素. 但与传统步行和地面交通等不同, 地铁 出行具有不堵车、速度快和上下车地点固定的特 点, 从数学上体现为明显的各向异性的距离度量 特性. 因此, 本文基于北京市城区的 POI 数据, 考 虑地铁出行对居民生活范围的拓宽效应, 提出了 “地铁生活圈”的概念, 以及基于地铁交通的距离 度量方法, 为生活圈的量化研究提供了思路. 通过 本文的研究成果, 可以更好地了解大数据下北京
市城区不同层次生活便利度指数住宅的空间分布, 也为城市规划管理者在优化平衡公共设施资源时 提供参考.

\section{2 数据来源与研究方法}

\section{1 数据来源}

POI 数据是地图服务的基础数据, 包括地点的 名称、地址、种类和地理坐标等信息. 本文获取高 德地图 2019 年 8 月的北京市城六区 POI 数据, 包 含东城区、西城区、海淀区、朝阳区、石景山区和 丰台区的 672217 个数据点, 分为 22 个 1 级大类和 235 个 2 级中类, 其中包括 33086 个住宅数据点. 因为高德 POI 数据点的经纬度坐标是在有偏移的 GCJ-02 坐标系下, 为了方便后续研究, 将其转化 为无偏移的 WGS-84 坐标系.

\section{2 生活便利度指数指标体系构建}

本文在相关研究 ${ }^{[11-12]}$ 的基础上，根据与住宅 居民日常生活密切相关的购物服务、教育科学、医 疗设施、交通设施、体育休闲、餐饮服务、生活服 务和金融服务 8 个方面, 构建北京市住宅的生活便 利度指数指标体系. 从高德 POI 数据的 235 个中类 中选取归纳出 24 类, 归总到这 8 个方面, 设置各 2 级指标的权重, 如表 1 所示.

\section{表 1 生活便利度指数指标体系}

\begin{tabular}{|c|c|c|}
\hline 1 级指标 & 2 级指标 & 指标权重 \\
\hline \multirow{4}{*}{ 购物服务 } & 商场 & 0.03 \\
\hline & 超级市场 & 0.05 \\
\hline & 综合市场 & 0.06 \\
\hline & 便利店 & 0.06 \\
\hline \multirow{2}{*}{ 教育科学 } & 学校 & 0.08 \\
\hline & 展馆 & 0.02 \\
\hline \multirow{4}{*}{ 医疗设施 } & 综合医院 & 0.01 \\
\hline & 专科医院 & 0.01 \\
\hline & 诊所 & 0.04 \\
\hline & 药店 & 0.04 \\
\hline \multirow{2}{*}{ 交通设施 } & 公交站 & 0.09 \\
\hline & 地铁站 & 0.06 \\
\hline \multirow{4}{*}{ 体育休闲 } & 影剧院 & 0.03 \\
\hline & 运动场馆 & 0.04 \\
\hline & 娱乐场馆 & 0.03 \\
\hline & 公园广场 & 0.05 \\
\hline \multirow{3}{*}{ 餐饮服务 } & 中餐厅 & 0.04 \\
\hline & 快餐厅 & 0.03 \\
\hline & 休闲餐饮 & 0.03 \\
\hline \multirow{3}{*}{ 生活服务 } & 物流和各类营业厅 & 0.04 \\
\hline & 家政维修服务 & 0.06 \\
\hline & 公共设施 & 0.05 \\
\hline \multirow{2}{*}{ 金融服务 } & ATM & 0.02 \\
\hline & 银行 & 0.03 \\
\hline
\end{tabular}




\section{3 生活便利度指数计算方法}

本文以 $X_{i, j}$ 表示住宅 $i$ 生活圈范围内第 $j$ 类公 共设施的数量. $X_{i, j}$ 不为 0 的数目越多, 表示住宅 生活圈范围内的公共设施越完备; $X_{i, j}$ 的值越大, 表示住宅周围的公共设施越丰富. 因为城市中建 筑物的阻挡，欧几里得度量不再适合 2 点间距离计 算. 由于北京市城区道路基本符合正东正南分布, 因此本文采用曼哈顿距离计算住宅与各公共设施 间的距离. 成年人的平均步行速度 $V_{1} \approx 5.5 \mathrm{~km} / \mathrm{h}$, 所以将 $15 \mathrm{~min}$ 和 $30 \mathrm{~min}$ 住宅生活圈的范围分别设 置为 $1375 \mathrm{~m}$ 和 $2750 \mathrm{~m}$.

POI 虽然具有数据点多、范围广和时效性强等 优点, 但其也有着低信息量的缺点, 即缺乏单个 POI 点规模、体量、从业人员等社会经济信息. 例 如, 在定量化研究中, 将所有运动场馆都视为相 同，忽视了不同运动场馆之间的体量差异，如国家 体育场和小区篮球场之间存在着很大差异。但同 类公共服务设施中个体之间的规模差异，可以体 现在 POI 的数量差异上, 在一定程度上弥补了这 种个体差异. 例如，一个大型商场包含多个餐饮和 娱乐类 POI 数据点, 而一个小商店只有 1 个 POI 数据点; 国家体育场包含多种体育设施类 POI 数 据点，小区篮球场只有 1 个 POI 数据点.

北京有着国内最发达的地铁系统，如图 1 所 示. 截至 2019 年 12 月, 北京地铁拥有 23 条运营线 路, 404 座运营车站和约 $699 \mathrm{~km}$ 的运营里程. 北京 地铁的工作日客运量均在 1000 万人次以上，在城 市居民的工作生活中发挥着重要的通行作用.

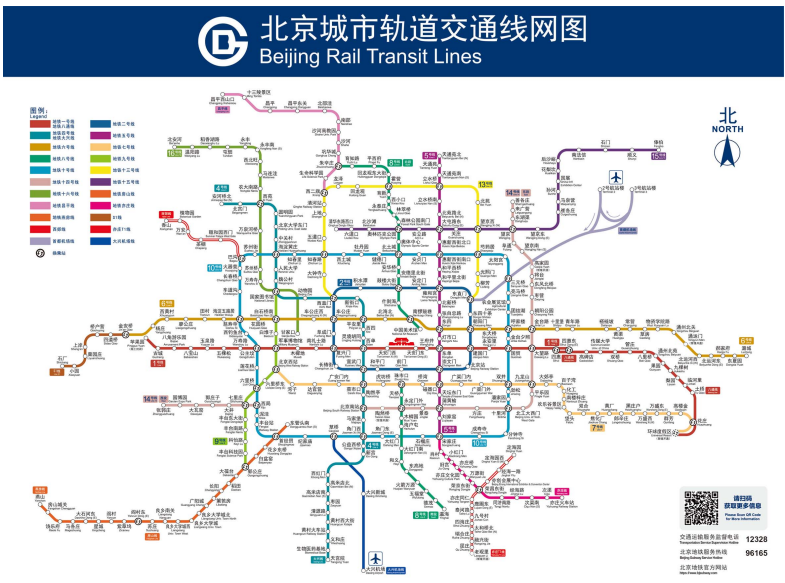

图 1 北京城市轨道交通线网图

地铁的出行扩大了人的出行范围，地铁沿线 的住宅生活便利程度高, 应当考虑地铁的作用. 北 京地铁运行的平均速度 $V_{2}$ 约为 $36 \mathrm{~km} / \mathrm{h}$, 乘客等待
地铁的平均时间为 $5 \mathrm{~min}$. 本文研究不同时间范围 下 (15 min 和 $30 \mathrm{~min}$ )步行和考虑地铁时的住宅生活 便利度指数.

在计算距离时, 如图 2 所示, 步行生活圈中, 2 点间距离 $D_{1}$ 即为 2 点间的曼哈顿距离, 即

$$
D_{1}=\left|X_{i}-X_{j}\right|+\left|Y_{i}-Y_{j}\right| \text {. }
$$

地铁生活圈中, 2 点间距离 $D_{2}$ 为 2 点分别与其 最近的地铁站间的曼哈顿距离、2 个地铁站间的乘 以系数 $c$ 的曼哈顿距离, 以及等待地铁期间的步行 可走距离 $S$ 之和，即

$$
\begin{aligned}
& D_{2}=\left|X_{i}-X_{i^{*}}\right|+\left|Y_{i}-Y_{i^{*}}\right|+\left|X_{j}-X_{j^{*}}\right|+ \\
& \quad\left|Y_{j}-Y_{j^{*}}\right|+\left(\left|X_{i^{*}}-X_{j^{*}}\right|+\left|Y_{i^{*}}-Y_{j^{*}}\right|\right) c+S .
\end{aligned}
$$

其中, $c=V_{1} / V_{2} ; S=V_{1} t, t$ 为平均等待时间.

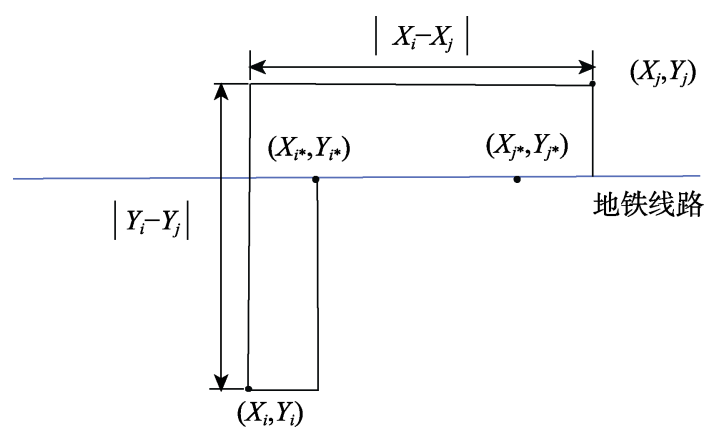

图 2 步行和地铁生活圈中距离计算图示

地铁的引人，使邻近地铁站的住宅的地铁生 活圈较步行生活圈范围更广. 如图 3 所示, 假设在 地铁线路上有平均间距为 $1500 \mathrm{~m}$ 的 $D_{1}, C_{1}, B_{1}, A$, $B_{2}, C_{2}$ 和 $D_{2}$ 地铁站, 存在位于地铁站 $A$ 处的住宅 $i$. 则其 $15 \mathrm{~min}$ 步行生活圈为以 $A$ 为中心的蓝色的 正方形范围, $15 \mathrm{~min}$ 地铁生活圈根据上文中 $D_{2}$ 距 离的计算, 为以 $A$ 为中心的蓝色正方形加上以 $D_{1}$, $C_{1}, B_{1}, B_{2}, C_{2}$ 和 $D_{2}$ 为中心的浅蓝色小正方形范 围. 可以看出, 邻近地铁线路住宅的地铁生活圈范 围较其步行生活圈范围有了明显的增加.

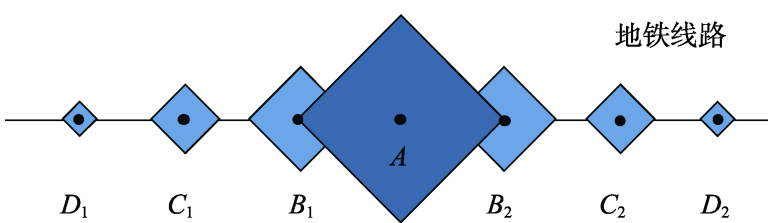

图 3 步行和地铁生活圈图示

在计算生活便利度指数时, 因为每个 POI 数 据 2 级指标的量纲不同, 无法直接线性相加, 需要 做归一化处理. POI 数据的分布为非负整数的非正 态分布，因而选择 Min-Max 的归一化处理方法. 
经过归一化处理后, 24 个 2 级 POI 指标均被放缩到 $0 \sim 1$, 并可以进行线性加权求和, 得到各个住宅的 生活便利度分数 $S_{i}=\sum_{j=1}^{24} X_{i, j}^{*} w_{j}$. 其中,

$$
X_{i, j}^{*}=\frac{X_{i, j}-\min \left(X_{*, j}\right)}{\max \left(X_{*, j}\right)-\min \left(X_{*, j}\right)} .
$$

最后将总分再次进行 Min-Max 归一化处理, 得到最终的住宅生活便利度指数. 依据指数高低 取 75\%, 50\%和 25\%为分界点, 将北京市住宅分为 生活便利度指数优质住宅、良好住宅、中等住宅和 待改进住宅; 得分越高, 表明住宅周围的服务设施 越多, 生活越便利. 研究不同层次住宅的空间分布, 可以为城市管理者的公共设施规划提供决策参考.

公交作为居民的常用交通出行方式，扩大了 居民的活动范围, 因此引人公交也可以提高评估 住宅生活便利度指数. 但公交和步行均属于地面 交通，基本符合各向同性的计算模型，本文的创新 点在于引人了各向异性的计算模型, 探索了地铁 这种特殊的交通出行方式对城市宜居的影响.

\section{3 研究结果对比与分析}

现有的研究对 POI 数据点间距离的计算方式 为欧几里得度量, 但因为城市中建筑物的阻挡, 人 只能沿道路方向行走，北京市内道路基本为正东 正南向，所以 2 点之间的距离使用曼哈顿距离计算 更为合适. 现有的对生活圈中住宅便利度指数研 究只考虑了居民步行可到达的范围, 并且限制在 $15 \mathrm{~min}$ 内. 但随着城市地铁的发展, 方便快速的地 铁拓宽了居民可到达的空间范围, 本文定量研究 了北京市城六区中住宅在 $15 \mathrm{~min}$ 和 $30 \mathrm{~min}$ 生活圈 考虑步行和地铁时的生活便利度指数, 分别得到 不同层次住宅的空间分布, 为城市规划提供决策 参考.

\section{1 生活便利度指数优质住宅空间分布特征} 对生活便利度指数优质住宅空间分布分析发 现, 如图 4 和图 5 所示, 优质住宅在整个城区中的 分布极不均衡, 4 种生活圈均集中分布在东城区、 西城区内，而在海淀区、石景山区、丰台区和朝阳 区的分布很少. 东城区和西城区的大部分区域都 为生活便利度指数优质小区，这是因为东城区和 西城区内有着数量丰富的商业中心、餐饮店、医院 和体育场馆等公共设施, 是北京市公共服务能力 最强的核心城区. 城市管理者在平衡区域功能, 增 添城市公共设施时应重点考虑其他区域.

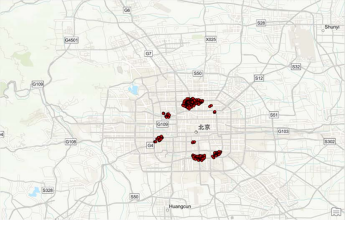

a. 15 min 步行生活圈

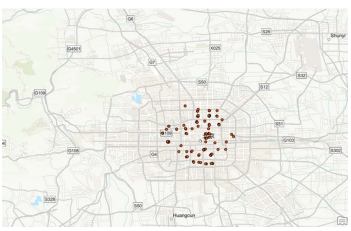

c. 15 min 地铁生活圈

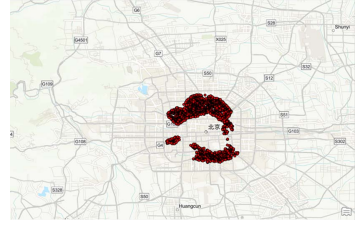

b. $30 \mathrm{~min}$ 步行生活圈

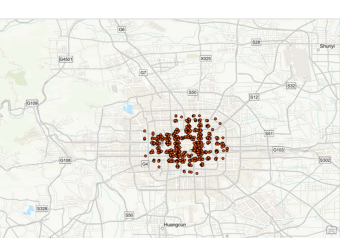

d. 30 min 地铁生活圈
图 4 优质住宅空间分布

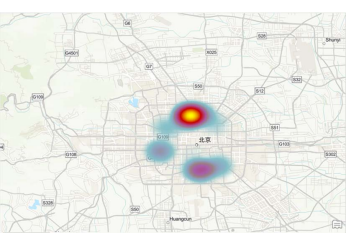

a. $15 \mathrm{~min}$ 步行生活圈

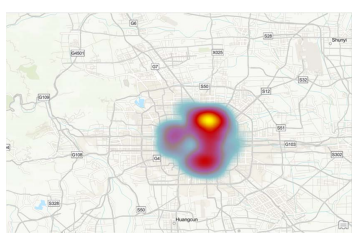

c. $15 \mathrm{~min}$ 地铁生活圈

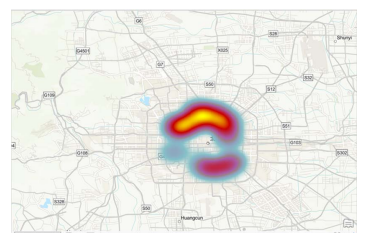

b. $30 \mathrm{~min}$ 步行生活圈

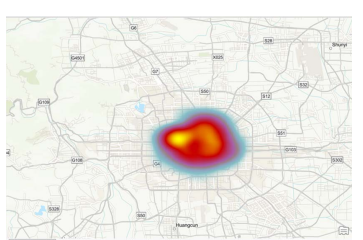

d. 30 min 地铁生活圈
图 5 优质住宅空间分布热力图

如图 6 所示, 生活便利度指数优质住宅在步行 和地铁生活圈中的空间分布有着明显的差别, 红 色点表示步行生活圈, 黄色点表示地铁生活圈. 步 行生活圈中优质住宅在空间上为连片集中分布, 主要集中在北二环与北三环之间, 以及南三环沿 线. $30 \mathrm{~min}$ 生活圈内的优质小区比 $15 \mathrm{~min}$ 生活圈要 多，这是因为随着时间的增加，住宅居民可到达的 公共服务设施也就更多. 地铁生活圈中优质住宅 在空间上为分散分布，主要集中在东城区和西城 区的各主要道路沿线, 特别是在地铁站附近.

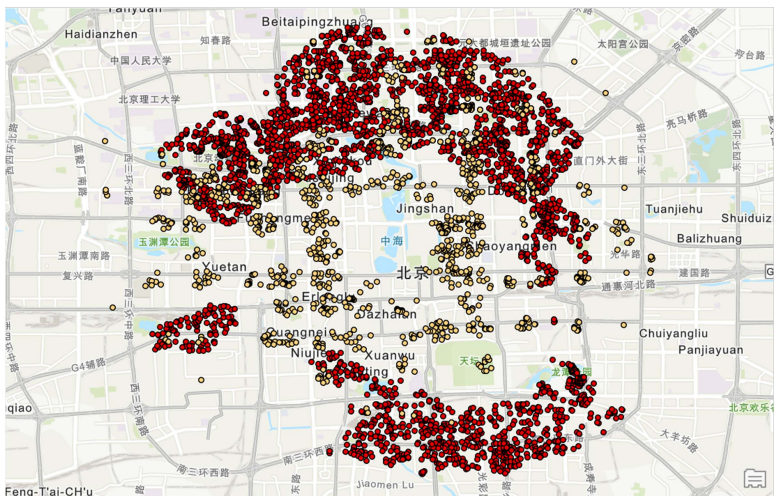

图 $630 \mathrm{~min}$ 生活圈优质住宅空间分布 
地铁作为特大城市大力发展的公共交通工 具, 极大拓展了城市居民的生活范围. 如图 7 和 图 8 所示的 $30 \mathrm{~min}$ 生活圈优质住宅热力图分布, 考虑地铁后，生活便利度指数优质住宅的空间分 布更为均匀，核心城区中均有优质住宅，更符合 现实情况.

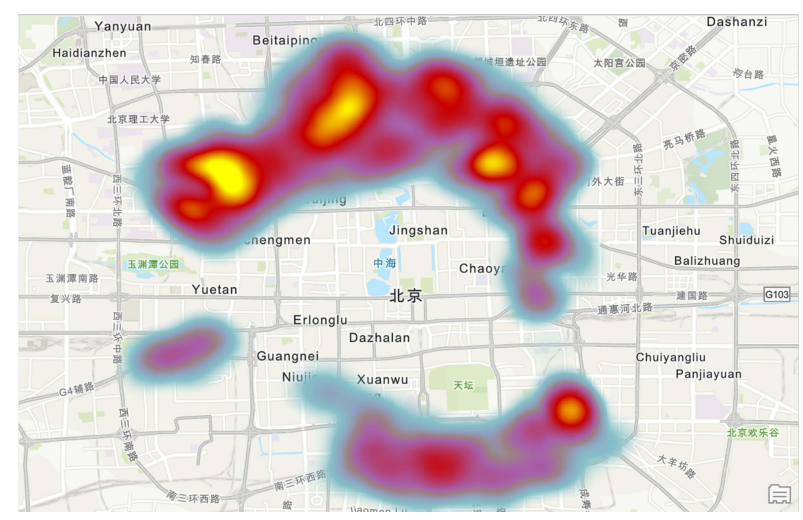

图 $730 \mathrm{~min}$ 步行生活圈优质住宅热力图分布

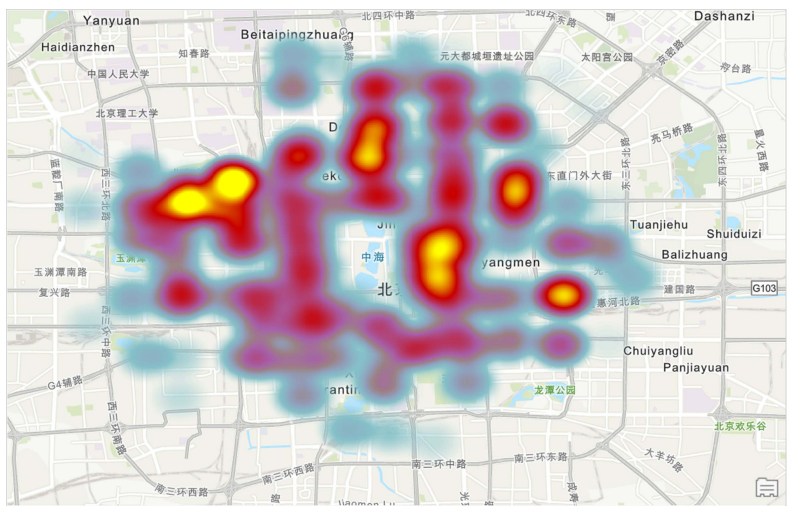

图 $830 \mathrm{~min}$ 地铁生活圈优质住宅热力图分布

\section{2 生活便利度指数良好和中等住宅空间分布 特征}

如图 9 所示, 生活便利度指数良好住宅与优质 住宅的空间分布相似，也是主要集中在东城区和 西城区. 步行生活圈中，良好住宅也是连片集中分 布，主要位于优质住宅区周围，分布于东三环沿 线、西三环沿线和长安街沿线. 地铁生活圈中, 生 活便利度指数良好住宅空间分布更为均匀合理, 主要分布在东西城区的主要道路沿线，以及北四 环、东四环和西四环沿线.

如图 10 所示, 生活便利度指数中等住宅主要 分布在城区次中心. 步行生活圈中, 中等住宅主要 分布在东西四环沿线、长安街四环外沿线、五道口、 亚运村和三元桥一带, 为北京市的次中心地带. 地 铁生活圈中，中等住宅的空间分布仍然呈现出在
市中心四环内均匀分布的特征, 从 $15 \mathrm{~min}$ 地铁生 活圈扩大到 $30 \mathrm{~min}$ 地铁生活圈后，中等住宅的范 围扩展到了四环与五环之间的道路沿线, 还包括 北五环外部分区域.

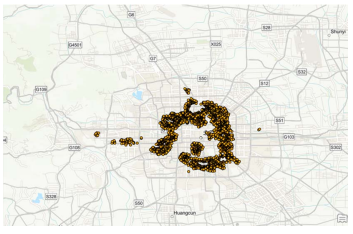

a. 15 min 步行生活圈

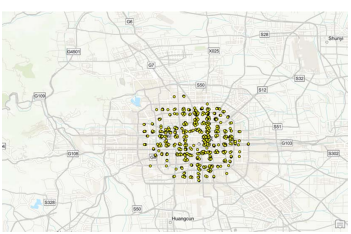

c. 15 min 地铁生活圈

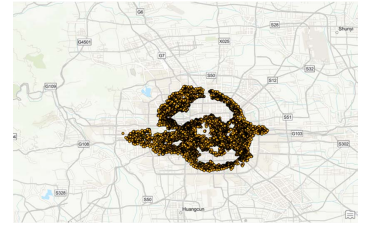

b. $30 \mathrm{~min}$ 步行生活圈

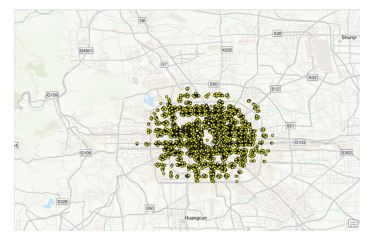

d. 30 min 地铁生活圈
图 9 良好住宅空间分布

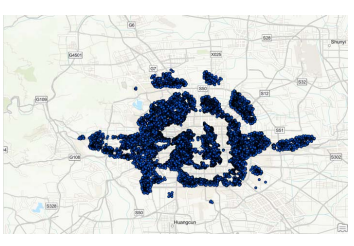

a. $15 \mathrm{~min}$ 步行生活圈

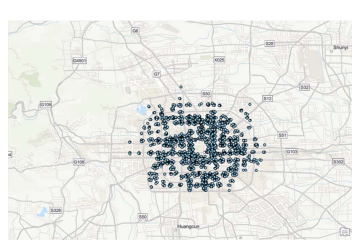

c. $15 \min$ 地铁生活圈

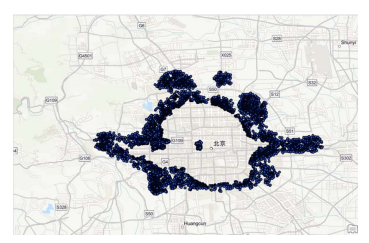

b. $30 \mathrm{~min}$ 步行生活圈

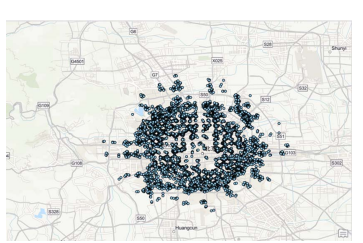

d. 30 min 地铁生活圈
图 10 中等住宅空间分布

\section{3 生活便利度指数待改进住宅空间分布特征}

生活便利度指数待改进住宅主要分布在五环 外，海淀区、石景山区、丰台区和朝阳区的住宅在 各生活圈中均主要为待改进住宅. 地铁拓宽了居 民的出行范围, 如图 11 所示, 地铁生活圈中待改 进住宅数量与步行生活圈相比均有着一定程度的 减少, 其中 $15 \mathrm{~min}$ 生活圈减少 $22.61 \%, 30 \mathrm{~min}$ 生活 圈减少 48.19\%. 2015 年国家统计局北京调查总队 发布的数据显示, 北京市五环外有 1098 万常住人 口, 占人口总数的 $51.10 \%$, 此外, 新增常住人口也 有向近郊区集聚的趋势. 随着这些区域人口的不 断增长, 公共设施的缺乏与居民日益增长的需要 之间的矛盾将越来越严峻, 因此城市管理者需要 在待改进住宅区域增加公共服务建设，提升居民 的生活质量, 实现各个区域的和谐平衡发展. 


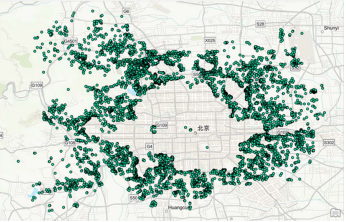

a. $15 \mathrm{~min}$ 步行生活圈

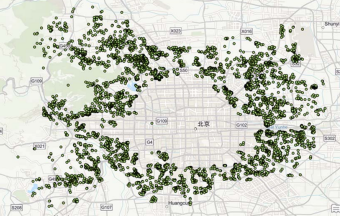

c. $15 \mathrm{~min}$ 地铁生活圈

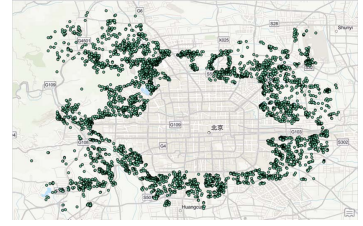

b. $30 \mathrm{~min}$ 步行生活圈

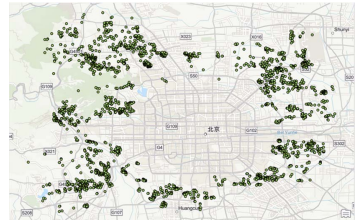

d. 30 min 地铁生活圈
图 11 待改进住宅空间分布

\section{4 公共服务设施达标率}

对于任一住宅，生活便利度指数的高低反映 了住宅居民对公共服务设施的可达性，影响了居 民的生活质量. 本文计算住宅生活便利度指数采 用的计算方式是分类计算，加权求和，但这种总体 的计算方式可能忽视了居民对各个大类公共服务 设施都存在的需要. 本文对于 “达标”的定义如下： 购物服务、教育科学、医疗设施、交通设施、体育 休闲、餐饮服务、生活服务和金融服务 8 大类下的 2 级分类分别至少存在 1 个; 如对于金融服务、 ATM 或银行至少存在 1 个, 则金融服务达标. 在本 文 4 种生活圈内，居民处于 $15 \mathrm{~min}$ 步行生活圈时 出行范围最小, 因此可获取到的公共服务设施最 少，此时的达标率需要得到特别关注

从表 2 中可以看出, 对于 15 min 步行生活圈 中的优质、良好和中等住宅，各类公共服务设施的 达标率都很高, 都为 $100.00 \%$. 观察优质、良好和 中等住宅的空间分布，可以看出北京市区核心区 域，特别是东城区和西城区，不仅拥有丰富的公共 服务设施资源，而且各项公共服务设施完备，在空 间上分布均匀合理. 而待改进住宅的各大类达标 率则较低，金融服务的达标率仅为 $85.12 \%$ ，说明

表 $215 \mathrm{~min}$ 步行生活圈各层次住宅 各公共服务设施达标率

\begin{tabular}{ccccc}
\hline 服务设施 & 优质 & 良好 & 中等 & 待改进 \\
\hline 购物 & 100.00 & 100.00 & 100.00 & 98.97 \\
教育 & 100.00 & 100.00 & 100.00 & 96.32 \\
医疗 & 100.00 & 100.00 & 100.00 & 96.75 \\
交通 & 100.00 & 100.00 & 100.00 & 99.09 \\
体育 & 100.00 & 100.00 & 100.00 & 98.33 \\
餐饮 & 100.00 & 100.00 & 100.00 & 99.62 \\
生活 & 100.00 & 100.00 & 100.00 & 99.63 \\
金融 & 100.00 & 100.00 & 99.98 & 85.12 \\
\hline
\end{tabular}

待改进住宅集中所在的五环外区域缺乏足够多的 银行或 ATM 机，金融部门应适当地增添相关设施. 待改进住宅的教育科学和医疗设施的达标率也较 低, 分别为 $96.32 \%$ 和 $96.75 \%$, 反映了待改进住宅 区域与居民生活水平密切相关的教育医疗资源不 足, 城市管理者在规划新的医院和学校时, 应当重 点考虑这些区域.

\section{4 结 语}

本文基于北京市城六区 672217 个全量 POI 数据点, 使用曼哈顿距离计算数据点间的距离, 对 住宅的生活便利度指数进行了定量化研究发现, 不同等级的住宅在空间分布上有着明显差异. 生 活便利度指数高的住宅主要集中在东城区和西城 区，它们作为核心城区拥有较多的各类公共服务 设施. 公共服务设施在城区中的不均匀分布，使步 行生活圈中生活便利度指数优质住宅在四环内集 聚连片分布. 北京拥有国内最发达的地铁系统, 地 铁在城市居民的生活出行中发挥着重要的作用, 有效地提升了沿线居民对各类公共服务设施的可 达性，使居民可以使用更多的公共服务设施，因此 地铁生活圈中优质住宅沿四环内主要道路分布, 空间分布更为合理. 2 种生活圈中待改进住宅都主 要集中在五环外, 而这些区域作为新增常住人口 集聚的主要区域, 亟需城市管理者加强公共服务 设施建设，为居民提供更为丰富的公共资源. 本文 的创新点在于, 提出面向地铁出行的各向异性距 离度量方法的计算模型, 探索了地铁这种特殊的 交通出行方式对城市居民生活便利度的影响.

\section{参考文献(References):}

[1] Zhang Jie, Lyu Jie. From mega-urban project design towards daily living space[J]. City Planning Review, 2003, 27(9): 40-45(in Chinese) (张杰，吕杰. 从大尺度城市设计到“日常生活空间”[J]. 城市 规划, 2003, 27(9): 40-45)

[2] Li Meng. The planning strategies of a 15-minute community life circle based on behaviors of residents[J]. Urban Planning Forum, 2017(1): 111-118(in Chinese) (李萌. 基于居民行为需求特征的“15 分钟社区生活圈”规划 对策研究 [J]. 城市规划学刊, 2017(1):111-118)

[3] Wu Kangmin, Zhang Hongou, Wang Yang, et al. Identify of the multiple types of commercial center in Guangzhou and its spatial pattern[J]. Progress in Geography, 2016, 35(8): 963-974(in Chinese)

(吴康敏, 张虹鸥, 王洋, 等. 广州市多类型商业中心识别与 
空间模式[J]. 地理科学进展, 2016, 35(8): 963-974)

[4] Lin Qing, Sun Fang, Wang Xiaomin, et al. Hierarchical system of Beijing commercial center judged from POI data[J]. Journal of Beijing Normal University: Natural Science, 2019, 55(3): 415-424(in Chinese)

(林清, 孙方, 王小敏, 等. 基于 POI 数据的北京市商业中心 地等级体系研究 [J]. 北京师范大学学报: 自然科学版, 2019, 55(3): 415-424)

[5] Xu Zening, Gao Xiaolu. A novel method for identifying the boundary of urban built-up areas with POI data[J]. Acta Geographica Sinica, 2016, 71(6): 928-939(in Chinese) (许泽宁, 高晓路. 基于电子地图兴趣点的城市建成区边界 识别方法[J]. 地理学报, 2016, 71(6): 928-939)

[6] Li Jiangsu, Liang Yan, Wang Xiaorui. Spatial clustering analysis of service industries in Zhengdong New District based on POI data[J]. Geographical Research, 2018, 37(1): 145-157(in Chinese)

(李江苏, 梁燕, 王晓萝. 基于 POI 数据的郑东新区服务业空 间聚类研究 [J]. 地理研究, 2018, 37(1): 145-157)

[7] Zhao Yundan, Shi Jing. Spatial distribution of public service facilities based on POI data-a case study of Hangzhou[J]. Territory \& Natural Resources Study, 2019(4): 36-37(in Chinese)

(赵云丹, 史静. 基于 POI 数据的杭州市中心城区公共服务 设施空间分布研究 [J]. 国土与自然资源研究, 2019(4): 36-37)

[8] Ding Juan, Li Junfeng. Spatial patterns of Chinese inbound tourist POI: an analysis of geographic information from Web
pictures[J]. Economic Geography, 2015, 35(6): 24-31(in Chinese)

(丁娟, 李俊峰. 基于 Web 地理图片的中国人境游客 POI 空 间格局[J]. 经济地理, 2015, 35(6): 24-31)

[9] Lu Y S, Huang J L. GLR: a graph-based latent representation model for successive POI recommendation[J]. Future Generation Computer Systems, 2020, 102:230-244

[10] Santos F, Almeida A, Martins C, et al. Using POI functionality and accessibility levels for delivering personalized tourism recommendations[J]. Computers, Environment and Urban Systems, 2019, 77: 101173

[11] Cui Zhenzhen, Huang Xiaochun, He Lianna, et al. Study on urban life convenience index based on POI data[J]. Geomatics World, 2016, 23(3): 27-33(in Chinese)

(崔真真, 黄晓春, 何莲娜, 等. 基于 POI 数据的城市生活便 利度指数研究 [J]. 地理信息世界, 2016, 23(3): 27-33)

[12] Zhao Yanyun, Zhang Bo, Zhou Fang. Research on the spatial measurement of in Beijing "15-minute community life circle" based on POI[J]. The World of Survey and Research, 2018(5): 17-24(in Chinese)

(赵彦云, 张波, 周芳. 基于 POI 的北京市“15 分钟社区生活 圈”空间测度研究[J]. 调研世界, 2018(5): 17-24)

[13] Zhang Bo, Zhao Yanyun, Zhou Fang. Research on the spatial cluster of the 15 minutes community life circle-based on POI data[J]. The World of Survey and Research, 2019(1): 49-56(in Chinese)

(张波, 赵彦云, 周芳. 小区“15 分钟社区生活圈”空间聚类 研究——基于 POI 数据[J]. 调研世界, 2019(1): 49-56) 